\title{
Transcriptomic analysis of radish (Raphanus sativus L.) spontaneous tumors
}

Tkachenko A.A. ${ }^{1,2}$, Dodueva I.E. ${ }^{1}$, Tvorogova V.E. ${ }^{1}$, Predeus A.V. ${ }^{3}$, Pravdina O.Yu. ${ }^{1}$, Kuznetsova K.A. ${ }^{1 *}$, Lutova L.A. ${ }^{1}$

${ }^{1}$ Department of Genetics and Biotechnology, Saint-Petersburg State University, St. Petersburg, Russia

${ }^{2}$ Laboratory of Computer Technologies, ITMO University, St. Petersburg, Russia

${ }^{3}$ Bioinformatics Institute, St. Petersburg, Russia

*email: kskuz95@mail.ru

Spontaneous tumorigenesis is a process that occur without any pathogen infection in different organs of various plant species. In higher plants, spontaneous tumors are uncommon other than pathogen-induced tumors, such as crown gall induced by Agrobacterium, and consequently less investigated. Several studies of spontaneous tumors in plants revealed that such tumors, as a rule, appear in plants with genotypes of mutants and interspecific hybrids. In radish, Raphanus sativus L., spontaneous tumors originate from the pericycle and cambium cells (Ilina et al., 2006; Lebedeva et al., 2015) which brings them closer to the lateral and adventitious roots. The inbred lines of radish (Raphanus sativus var. Radicula Pers) develop spontaneous tumors on the taproot during the flowering period. Among these lines, the connection between the spontaneous tumor development and the altered balance of the main phytohormones, as well as ectopic expression of meristem-specific genes, was previously demonstrated. In this work, we analyzed the differential gene expression in the radish spontaneous tumors versus the lateral roots using the RNA-seq method. Analysis of GO categories revealed that most enriched upregulated pathways in spontaneous tumors were associated with cell division and expansion, especially genes that regulate G2-M transition and cytokinesis. Among downregulated pathways, the ones involved in stress and wounding response, as well as in the production of JA and glucosinolates, were overrepresented. Our data may help to elucidate the mechanisms of spontaneous tumor development in higher plants.

Acknowledgements: This work was supported of the Ministry of Science and Higher Education of the Russian Federation in accordance with agreement No. 075-15-2020922 date 16.11.2020 on providing a grant in the form of subsidies from the Federal budget of Russian Federation. The grant was provided for state support for the creation and development of a World-class Scientific Center "Agrotechnologies for the Future". 